\title{
T cells take directions from supporting cast in graft-versus-host disease
}

\section{Derk Amsen}

Department of Hematopoiesis, Sanquin Research and Landsteiner Laboratory for Blood Cell Research, Amsterdam, Netherlands.

\author{
Allogeneic hematopoietic stem cell transplantation (HSCT) remains the \\ only treatment option for several severe hematological malignancies. The \\ development of graft-versus-host disease (CVHD) is a common complication \\ of the procedure and results when donor T cells become activated against \\ recipient-specific antigens. The factors that drive the alloreactive $T$ cell \\ response are not completely understood. In this issue of the $\mathrm{JCl}$, Chung \\ and colleagues present evidence that stromal cells within lymphoid tissue \\ express the Notch ligands Delta-like 1/4 (DLL1 and DLL4), which in turn \\ directly activate T cells. Importantly, inhibition of DLL1/DLL4-mediated \\ Notch signaling in murine HSCT models dramatically reduced GVHD and \\ improved graft survival.
}

\section{T cells in allogeneic hematopoietic stem cell transplantation}

$T$ cells act as a double-edged sword in allogeneic hematopoietic stem cell transplantation (HSCT), the only available curative option for many hematological malignancies (1). Alloreactive $\mathrm{T}$ cells within the graft help eradicate malignant clones, but unfortunately also attack healthy tissues of the recipient. The resulting graftversus-host disease (GVHD) is fatal in approximately $15 \%$ of transplant patients, with close to $90 \%$ mortality in patients who fail to respond to immunosuppression (1). Better treatment options are needed and would ideally suppress GVHD, while preserving graft-versus-leukemia (GVL) reactivity. A greater understanding of how alloreactive $\mathrm{T}$ cell responses are regulated is key to finding more effective treatments. In this issue, Chung et al. reveal that the Notch signaling pathway is one factor that controls alloreactive $\mathrm{T}$ cell responses (2).

Notch is a cell-surface receptor that responds to membrane-bound ligands of the jagged and delta-like (DLL) families (3) and is expressed on a variety of cells, including T cells. Notch is known to regulate T cell functions and differentiation; however, this receptor is not universally required for all $\mathrm{T}$ cell responses (3). Previous work has shown that inhibition of Notch signaling after allogeneic bone marrow transplantation in mice impedes the differentiation of alloreactive $\mathrm{T}$ cells into cytokine-producing effector cells, consequently preventing the development of GVHD, while still allowing GVL activity (4). Chung et al. wanted to better understand the generation of the alloreactive $\mathrm{T}$ cell response and therefore searched for the cellular origin of the ligands that activate Notch in alloreactive $\mathrm{T}$ cells (2). This search led them to make a surprising discovery.

\section{Lymphoid tissue stromal cells}

GVHD is caused by naive T cells (1), which have never before been activated by antigen and require specific signals to be fully aroused from their somnolent state. In contrast, the GVL response relies on crossreactive memory $\mathrm{T}$ cells, which have different activation requirements (1). Accord-

Related Article: p. 1574

Conflict of interest: The author has declared that no conflict of interest exists

Reference information: / Clin Invest. 2017;127(4):1215-1217. https://doi.org/10.1172/JCI93552.

ing to immunological canon, the ability to awaken naive $\mathrm{T}$ cells belongs to the realm of DCs, informally crowned by immunologists with the epithet "professional antigen-presenting cells (APCs)" (5). DCs pick up antigens in peripheral tissues to present to naive $\mathrm{T}$ cells in secondary lymphoid organs (SLOs). Additionally, DCs deliver a broad repertoire of costimulatory signals, which instruct naive $\mathrm{T}$ cells to proliferate and acquire the ability to produce effector molecules such as cytokines and/or cytolytic molecules. The expression of costimulatory molecules is induced by recognition of microbes, a feature that presumably ensures that full-blown $\mathrm{T}$ cell responses are induced only when needed (6). Ligands for Notch are among the costimulatory molecules expressed by DCs (3). Surprisingly, Chung et al. found that the Notch-activating signal that enables the $\mathrm{T}$ cell response in GVHD does not come from DCs. Instead, their data indicate that this signal is provided by a CCL19-producing lymphoid tissue stromal cell (2).

SLOs, such as lymph nodes and the splenic white pulp, are swarming with white blood cells, and at least five stromal cell populations are responsible for the organization of these structures (7). The best-known CCL19-producing stromal cells are fibroblastic reticular cells (FRCs), which use this chemokine (together with CCL21) to attract $\mathrm{T}$ cells and DCs into so-called "T cell areas" (Figure 1). FRCs form a 3D road system for the migration and interaction of $\mathrm{T}$ cells and DCs. FRCs also create conduits that allow antigens in the lymph to penetrate into SLOs and support T cell viability by producing IL-7 (7). Given the intimate interaction between FRCs and $\mathrm{T}$ cells, it is perhaps not surprising that these stromal cells influence $\mathrm{T}$ cells beyond a mere supporting role. Indeed, FRCs can induce $\mathrm{T}$ cell tolerance to self-antigens and prevent excessive $\mathrm{T}$ cell responses by the production of nitric oxide (7). The current work by Chung and colleagues now suggests that these FRCs, 


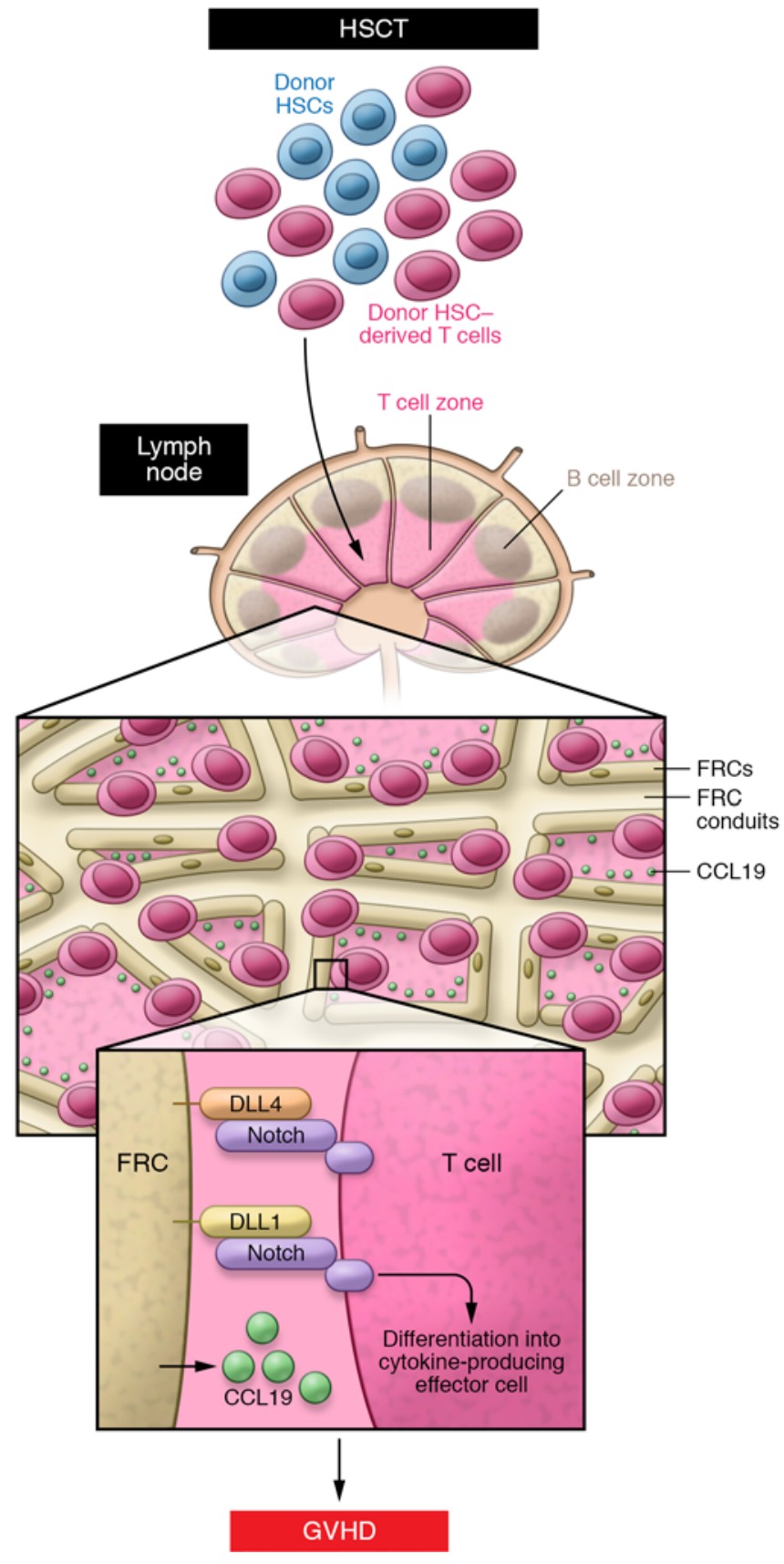

unexpectedly, also provide signals that actively promote $\mathrm{T}$ cell responses via activation of Notch (2).

A note of caution regarding this conclusion: the study by Chung et al. does not unequivocally identify FRCs as the relevant source of T cell-activating Notch ligands. The authors evaluated mice harboring deletions of the genes encoding DLL1 and DLL4, the two Notch ligands responsible for the GVHD-associated $\mathrm{T}$ cell reaction (8), specifically in cells expressing a Ccl19Cre transgene. Although FRCs are major producers of CCL19 (7), this transgene was also expressed in other stromal cell types (2). These included follicular DCs (FDCs), which, despite their name, are not related to DCs, and lymphatic endothelial cells (LECs). All three of these stromal cell types also expressed DLL4. Each of these could therefore theoretically be responsible for the nefarious Notch signal that fuels the GVHD-inducing $\mathrm{T}$ cell response.

The finding that a stromal cell is responsible for delivering activating signals to $\mathrm{T}$ cells is provocative, even though the precise identity of the cell type involved may still be uncertain. An obvious question
Figure 1. Stromal cells directly activate Notch on allogeneic $\mathrm{T}$ cells. FRCs within SLOs, such as lymph nodes, are located in the T cell zone, where they help form a fibrous network within the tissue. FRCs also produce the T cell chemoattractant CCL19, which is an important mediator of T cell trafficking. In this issue, Chung et al. present evidence that during HSCT, Cc/19-expressing stromal cells express the Notch ligands DLL1 and DLL4. DLL1/DLL4-mediated Notch signaling directly promotes the activation of alloreactive T cells, resulting in GVHD. Given their location in the T cell zone, FRCs are most likely the Cc/19-expressing cell responsible for the activation of Notch in alloreactive T cells; however, other stromal cells, including FDCs and LECs, also express Cc/19 and Notch ligands. Under normal conditions, FDCs and LECs are not likely to encounter $T$ cells, but this possibility cannot be entirely ruled out.

is whether this role for stromal cells is a peculiarity of allogeneic T cell transplantation. Indeed, DCs are apparently dispensable for GVHD development in this setting $(9,10)$, in sharp contrast to their central role in the generation of antimicrobial $\mathrm{T}$ cell responses. Moreover, the highest expression of DLL4 was found on the surface of FDCs (2), which reside in B cell areas not normally accessed by most $\mathrm{T}$ cells. Therefore, if this high expression identifies FDCs as the source of the Notch-activating signal, their interaction with $\mathrm{T}$ cells would presumably require abnormal $\mathrm{T}$ cell trafficking. This could perhaps occur after $\mathrm{T}$ cell transfer into irradiated hosts. On the other hand, surface levels of Notch ligands do not correlate well with activity (11), and evidence exists that Notch ligands on lymphoid tissue stromal cells can affect $\mathrm{CD} 4^{+}$ $\mathrm{T}$ cells also in normal mice (i.e., without irradiation) (12). A recent study showed that inactivation of the receptor for lymphotoxin $\beta$ (Lt $\beta$ ) in stromal cells prevented effective T cell-mediated responses to several viruses in mice (13). Neither conduit function nor recruitment of T cells and DCs was perturbed, suggesting that $\operatorname{Lt} \beta$ induces another property in stromal cells necessary for optimal $\mathrm{T}$ cell responses to microbial pathogens. The results by Chung et al. suggest that this property might involve the expression of activating signals, such as DLL molecules (2). Notch promotes the differentiation of effector $\mathrm{T}$ cells but is not overtly required for the differentiation of memory or regulatory $\mathrm{T}$ cells $(3,14,15)$. An exciting hypothesis based on the find- 
ings of Chung et al. could be that different microanatomical niches exist that physically separate the induction of $\mathrm{T}$ cell fates on the basis of whether the local stromal cells express ligands for Notch.

\section{How does Notch do it?}

Although the current work by Chung et al. relied on the elimination of Notch ligands (2), another study showed that direct inhibition of Notch signaling in $\mathrm{T}$ cells also reduces GVHD, documenting a $\mathrm{T}$ cellintrinsic function (16). It is not clear exactly how Notch controls the $\mathrm{T}$ cell response in GVHD, but multiple mechanisms are probably involved. Inhibition of Notch has little effect on $\mathrm{T}$ cell expansion, but does diminish the acquisition of effector functions $(4,8,16)$. This latter finding fits with studies showing that Notch directly regulates genes that encode transcription factors such as T-bet, ROR $\gamma \mathrm{t}$, and GATA3, which control effector differentiation, and genes that encode effector proteins such as IL-4, IFN- $\gamma$, IL-17, and granzyme B (3). Additionally, Notch indirectly promotes $\mathrm{T}$ cell responses by diminishing the suppressive capacity of Tregs (17) and expansion of these suppressive cells, especially after allogeneic $\mathrm{T}$ cell transfer $(4,8,16)$. Tregs are potent suppressors of GVHD, and adoptive cellular therapy with such cells is currently being examined as a therapeutic option (1).

\section{Clinical applicability}

What do the findings by Chung et al. (2) mean for patients? A critical question will be whether Notch has a similar role in human GVHD. Future studies should also evaluate whether inhibition of Notch also prevents the development of chronic GVHD, which is different in nature than acute GVHD and is the main cause of mortality associated with allogeneic HSCT (1). There is some ground for optimism that targeting Notch will prove to be beneficial in limiting chronic GVHD. Activation of alloreactive $\mathrm{T}$ cells in the absence of Notch signaling resulted in a state of nonresponsiveness, thereby decreasing the likelihood that they will develop pathogenic function later on. Moreover, inhibition of Notch resulted in expansion of the Treg repertoire $(4,8,16)$, which may also sup- press the future development of chronic GVHD. Finally, acute GVHD is one of the greatest risk factors for the development of chronic GVHD (1). Preventing the acute form might therefore also protect against chronic disease.

How attractive is Notch, then, as a therapeutic target? At face value, the widespread role of Notch signaling in physiological processes seems to make this pathway less than ideal for therapeutic exploitation. Prolonged general inhibition of Notch signaling does indeed yield undesirable side effects, including intestinal toxicity and vascular problems $(18,19)$. Nonetheless, promising leads to circumvent these side effects do exist. Notch receptors respond to at least five different ligands of the jagged and DLL families, and separate functions of Notch depend on different ligands (3). Antibody-mediated blockade of DLL1 and DLL4 is sufficient to prevent GVHD, leaving the jagged-dependent functions of Notch untouched (8). Importantly, Chung et al. now show that the critical role of Notch in the development of GVHD is limited to the first days after $\mathrm{T}$ cell transfer (2), such that a single injection of blocking antibodies against DLL1 and DLL4 is sufficient to avert pathology in animal models. Such short-term inhibition is much less likely than prolonged inhibition to cause side effects. Clinical trials exploring the therapeutic potency of Notch inhibition for the treatment of GVHD have been initiated (1). These findings by Chung et al. suggest that testing prophylactic shortterm inhibition of Notch, preferably with reagents that block DLL1 and DLL4, might be worthwhile.

\section{Acknowledgments}

The author thanks Rene van Lier (Sanquin Research Amsterdam, Netherlands) and Arjan Lankester (Leiden University Medical Center, Leiden, Netherlands) for critical reading of the manuscript.

Address correspondence to: Derk Amsen, Department of Hematopoiesis, Sanquin Research and Landsteiner Laboratory for Blood Cell Research, Plesmanlaan 125, 1066CX, Amsterdam, Netherlands. Phone: 31.20.512.3976; E-mail: d.amsen@ sanquin.nl.
1. Blazar BR, Murphy WJ, Abedi M. Advances in graft-versus-host disease biology and therapy. Nat Rev Immunol. 2012;12(6):443-458.

2. Chung J, et al. Fibroblastic niches prime T cell alloimmunity through Delta-like Notch ligands. JClin Invest. 2017;127(4): 1574-1588.

3. Amsen D, Helbig C, Backer RA. Notch in T cell differentiation: All things considered. Trends Immunol. 2015;36(12):802-814.

4. Zhang Y, et al. Notch signaling is a critical regulator of allogeneic $\mathrm{CD} 4{ }^{+} \mathrm{T}$-cell responses mediating graft-versus-host disease. Blood. 2011;117(1):299-308.

5. Steinman RM. Decisions about dendritic cells: past, present, and future. Annu Rev Immunol. 2012;30:1-22.

6. Iwasaki A, Medzhitov R. Toll-like receptor control of the adaptive immune responses. Nat Immunol. 2004;5(10):987-995.

7. Fletcher AL, Acton SE, Knoblich K. Lymph node fibroblastic reticular cells in health and disease. Nat Rev Immunol. 2015;15(6):350-361.

8. Tran IT, et al. Blockade of individual Notch ligands and receptors controls graft-versus-host disease. J Clin Invest. 2013;123(4):1590-1604.

9. Koyama M, et al. Recipient nonhematopoietic antigen-presenting cells are sufficient to induce lethal acute graft-versus-host disease. Nat Med. 2011;18(1):135-142.

10. Toubai $\mathrm{T}$, et al. Induction of acute GVHD by sex-mismatched $\mathrm{H}-\mathrm{Y}$ antigens in the absence of functional radiosensitive host hematopoietic-derived antigen-presenting cells. Blood. 2012;119(16):3844-3853.

11. Koo BK, et al. Mind bomb 1 is essential for generating functional Notch ligands to activate Notch. Development. 2005;132(15):3459-3470.

12. Fasnacht $\mathrm{N}$, et al. Specific fibroblastic niches in secondary lymphoid organs orchestrate distinct Notch-regulated immune responses. J Exp Med. 2014;211(11):2265-2279.

13. Chai Q, et al. Maturation of lymph node fibroblastic reticular cells from myofibroblastic precursors is critical for antiviral immunity. Immunity. 2013;38(5):1013-1024.

14. Backer RA, et al. A central role for Notch in effector CD8(+) T cell differentiation. Nat Immunol. 2014;15(12):1143-1151.

15. Mathieu M, Duval F, Daudelin JF, Labrecque N. The Notch signaling pathway controls shortlived effector $\mathrm{CD}^{+} \mathrm{T}$ cell differentiation but is dispensable for memory generation. J Immunol. 2015;194(12):5654-5662.

16. Sandy AR, et al. T cell-specific notch inhibition blocks graft-versus-host disease by inducing a hyporesponsive program in alloreactive $\mathrm{CD} 4^{+}$and CD8 ${ }^{+}$T cells. J Immunol. 2013;190(11):5818-5828.

17. Charbonnier LM, Wang S, Georgiev P, Sefik E, Chatila TA. Control of peripheral tolerance by regulatory T cell-intrinsic Notch signaling. Nat Immunol. 2015;16(11):1162-1173.

18. Garber K. Notch emerges as new cancer drug target. J Natl Cancer Inst. 2007;99(17):1284-1285.

19. Yan M. Therapeutic promise and challenges of targeting DLL4/NOTCH1. Vasc Cell. 2011;3:17. 